\title{
Das Pasticcio in den böhmischen Ländern in der ersten Hälfte des 18. Jahrhunderts
}

Jana Spáčilová

\begin{abstract}
Die Studie befasst sich mit dem Pasticcio, einer bedeutenden Untergattung der italienischen Opera seria im 18. Jahrhundert. Sie erörtert die strukturellen Prinzipien der Barockoper als Voraussetzungen für die Entstehung des Pasticcios und erläutert dessen definitorischen Rahmen. Als Beispiel für die damalige Verbreitung des Pasticcios wurden die Länder der böhmischen Krone mit ihren unterschiedlichen institutionellen Bedingungen (städtisches versus privates aristokratisches Theater) gewählt. Auf Basis der erhaltenen Libretti und Partituren lassen sich im Untersuchungsgebiet verschiedene Pasticcio-Formen differenzieren, Erweiterungen hin zum Oratorium und Ordenstheater eingeschlossen.
\end{abstract}

\section{Schlüsselwörter}

Oper, Oratorium, Barockmusik, Pasticcio, Arie, böhmische Länder

\begin{abstract}
The study deals with the pasticcio, a significant sub-genre of the Italian opera seria in the 18th century. It discusses the structural principles of the baroque opera as prerequisites for the creation of the pasticcio and explains its defining framework. The countries of the Czech Crown with their different institutional conditions (municipal versus private aristocratic theatres) were chosen as an example of the spread of pasticcios at the time. On the basis of the librettos and scores obtained, different forms of pasticcio can be differentiated in the studied area, including extensions to the oratorio and religious theatre.
\end{abstract}

\section{Keywords}

Opera, Oratorio, Baroque music, pasticcio, aria, Czech lands

Die vorgelegte Studie ist ein Ergebnis des Forschungsprojekts Die Reflexion der italienischen Musikkultur in den böhmischen Ländern des Barock, finanziert durch die Philosophische Fakultät der Palacký-Universität in Olmütz (FPVC2019/10). 
Hier hörete ich verschiedene Opern, die aber alle von Arien verschiedener Meister zusammen geflicket waren, welche Art von Einrichtung die Welschen eine Pastete, (un pasticcio) zu nennen pflegen.

(QUANTZ 1755: 230)

In diesem Zitat wurde vermutlich erstmals der Terminus ,Pasticcio“ in Bezug auf die italienische Barockoper verwendet. Es handelt sich um eine Bezeichnung des deutschen Flötenspielers, Komponisten und Musiktheoretikers Johann Joachim Quantz für Opern, die er in den Zwanzigerjahren des 18. Jahrhunderts in Italien kennengelernt hatte. ${ }^{1}$ In der Auffassung von Quantz besitzt diese Bezeichnung (schon allein aufgrund der Verwendung des Worts ,flicken') einen pejorativen Beigeschmack, der im Laufe der zweiten Hälfte des 18. Jahrhunderts mit der Etablierung des Konzepts eines unantastbaren, von einem genialen Komponisten geschaffenen Werks noch deutlicher wurde. Die heutige Musikwissenschaft hält das Pasticcio für einen weniger wichtigen Zweig der italienischen Oper von nur mittelmäßigem ästhetischen Wert.

Doch bei einer gründlicheren Auseinandersetzung mit der Oper des 18. Jahrhunderts kommt man zu der Feststellung, dass das Pasticcio-Prinzip viel verbreiteter war als bislang vermutet, und zwar sowohl hinsichtlich des Entstehens neuer musikdramatischer Werke als auch hinsichtlich der Aufführungspraxis. Insbesondere im Kontext der Rezeption der italienischen Oper jenseits der Alpen spielte dieses Prinzip eine Schlüsselrolle bei der Etablierung einer neuen Gattung. Deswegen ist es auch notwendig, ihm mehr Aufmerksamkeit zu widmen. Im Folgenden wird versucht, die Umsetzung dieses Prinzips auf dem Gebiet der böhmischen Länder - seit den Zwanzigerjahren des 18. Jahrhunderts eines der bedeutendsten Zentren der italienischen Oper - zu verfolgen.

\section{Voraussetzungen: das strukturelle Prinzip der italienischen Oper des 18. Jahrhunderts}

Bevor man seine Aufmerksamkeit der Erläuterung des Pasticcios und seiner Umsetzung im ausgewählten Zeitraum zuwendet, ist es notwendig, über die Gründe, die zu seiner Entstehung führten, nachzudenken. Die Grundvoraussetzung, die die massenhafte Ausbreitung des Pasticcio-Prinzips möglich machte, ist der eigentliche strukturelle Aufbau der italienischen Oper im Barock. Schon seit dem Entstehen der Gattung um das Jahr 1600 war für die Oper die Verbindung des Worts und der Musik bzw. des rezitativen und arienmäßigen Prinzips charakteristisch. Das erste Prinzip basiert auf der Rezitation des vertonten Texts, wohingegen beim anderen Prinzip eher die musikalische Komponente im Vordergrund steht.

1 Konkret waren es die um die Jahreswende 1725/1726 in Florenz aufgeführten Opern L'Ingratitudine gastigata, La Mariane, Farnace und Forza del Sangue, in deren Libretti die Komponistennamen nicht angegeben wurden. Vgl. (SARTORI 1990-1994, Nr. 13213, 14823, 9733 und 10842). 
Im Laufe der Gattungsentwicklung entfernten sich beide Prinzipien voneinander, was sich sowohl auf der Textebene (freier Vers versus gebundener Vers) ${ }^{2}$ als auch auf der Ebene der Musik zeigte (Rezitative wurden einfach syllabisch vertont und mit einer Continuo-Gruppe begleitet, während bei Arien das Orchester zum Einsatz kam). ${ }^{3}$ Ein wichtiges Unterscheidungsmerkmal besteht auch in der Durchbrechung der Interaktion zwischen den Beteiligten der Opernvorstellung - während in den Rezitativen die Figuren miteinander kommunizieren, bietet die Arie Gelegenheit, sich an den Zuschauer zu wenden. Die Individualisierung und die Trennung des rezitativen und arienmäßigen Prinzips ließen die sogenannte ,Nummernoper entstehen, die sich aus deutlich begrenzten, durch Rezitative verbundenen Musiknummern zusammensetzte. Die zeitliche Ausdehnung von Rezitativen und Arien wurde in der zweiten Hälfte des 18. Jahrhunderts Ziel berechtigter Kritik und führte zu Reformbestrebungen. Auch heute gilt der zeitliche Umfang der Vorstellung als Ursache dafür, dass das Publikum Barockopern in einer nicht gekürzten Form nur schwer aufzunehmen bereit ist. ${ }^{4}$

Vereinfacht ist die Struktur der Barockoper so zu beschreiben, dass das Rezitativ die Handlung nach vorne treibt, während die Arie diese reflektiert. Raum für eine Arie gab es regelmäßig gegen Ende der Szene, wo der Figur vor dem Verlassen der Bühne die Gelegenheit eingeräumt wird, sich in einem Kommentar zu äußern oder ihren aktuellen psychischen Zustand zu beschreiben. Und gerade hier kommt es zu einem Schlüsselmoment: Weil die Skala der Affekte (im Sinne von Gefühlsregungen) nicht unendlich ist, kristallisierten sich mehrere Typen von Arien mit einem gleichen oder ähnlichen Charakter heraus. ${ }^{5}$ Die Texte dieser typisierten Arien waren möglichst allgemein gehalten und enthielten mit wenigen Ausnahmen weder eine Anrede noch Bezüge zu konkreten Umständen der Handlung. Mit der Auflösung der Verbindung der Arie zu einer konkreten Handlungssituation wurde es in der Praxis möglich, Arien auszuleihen, sodass in einem ähnlichen dramatischen Kontext eine zu derselben Kategorie gehörende Arie zu Gehör gebracht werden konnte. Die Austauschbarkeit von Arien war auch aus musikalischen Gründen gegeben. Zwischen dem Rezitativ und der Arie gab es zwar ein tonales Verhältnis (entweder auf Basis der Dominant- oder der Terzverwandtschaft), für die Integration eines neuen Stücks in einer anderen Tonart reichte es jedoch, lediglich die abschließende Modulation des vorherigen Rezitativs geringfügig zu modifizieren oder die Arie zu transponieren, ohne dass das Ganze irgendwie grundlegend beeinträchtigt worden wäre.

2 Für das Rezitativ ist ein syllabotonischer Vers mit ungerader Silbenzahl (,verso sciolto ${ }^{\circ}$ ) typisch, wobei der Reim nur an den Kulminationspunkten des Dramas und am Ende der Szene vorkommt. Die Arie ist demgegenüber gereimt und weist eine abweichende Versstruktur auf.

3 Beim Rezitativ konnte allerdings bei speziellen Gelegenheiten auch das Orchester begleiten (,recitativo accompagnato'), während Arien natürlich auch mit einem Basso continuo (d.h. Cembalo und ein Streichinstrument, am häufigsten Violoncello) auskommen konnten.

4 Da die Aufführungszeit einer Barockoper ohne Pause dreieinhalb bis vier Stunden dauert, nehmen auch spezialisierte Ensembles oft zahlreiche Streichungen vor, und zwar insbesondere bei den Rezitativen.

5 Das Prinzip der Typisierung wurde in der Barockoper zum Beispiel auch auf der Ebene der Gestaltung des Bühnenbilds realisiert, vgl. (SPÁČILOVÁ 2011). 
Dieses Prinzip der Unabhängigkeit von Arien von der Handlung eines Stücks wurde zum wichtigsten strukturellen Merkmal der Barockoper. Angesichts des zeitgenössischen legeren Umgangs mit Urheberrechten kam es dabei nicht darauf an, wer der Autor der neu eingelegten Teile war. Als natürliche Konsequenz einer solchen Entwicklung entstand ein musikdramatisches Stück, in dem die Musik verschiedener Komponisten verwirklicht wurde, also ein Pasticcio.

\section{Definitionen: das Pasticcio und seine Varianten}

Nach ersten Vorüberlegungen von Gordana Lazarevich wurde das barocke Opern-Pasticcio erst in den letzten Jahren wieder zum Gegenstand theoretischer Reflexionen (LAZAREVICH 1976). ${ }^{6}$ Die wichtigsten Impulse gingen dabei von Forschern aus, die sich mit Georg Friedrich Händel und neuerdings auch mit Antonio Vivaldi beschäftigten, in deren Opernwerk Pasticci eine bedeutende Rolle spielten (STROHM 1985, 2008). Angesichts der häufigen Verwendung dieses Fachbegriffs muss sich die Musikwissenschaft allerdings damit auseinandersetzen, dass die Bedeutung des Begriffs nicht genau bestimmt ist (SIEGERT 2016). Handelt es sich um eine eigene Musikgattung oder eher nur um eine Form der zeitgenössischen Aufführungspraxis? Was sind die charakteristischen Merkmale eines Opernwerks, die dieses haben muss, damit man es Pasticcio nennen kann? Welche Erscheinungen sind eher im Grenzbereich angesiedelt und wann kann man nicht mehr von einem Pasticcio sprechen?

Vorweg ist festzuhalten, dass wir es im Fall des Pasticcios, und insbesondere seiner ästhetischen Bewertung, zum größeren Teil mit neuzeitlichen, also nicht-historischen Konzepten zu tun haben. Der Begriff wurde schon bei der ersten Auseinandersetzung mit dem Phänomen geprägt - in den Bemerkungen eines deutschen Theoretikers aus dem Jahre 1755 darüber, wie dreißig Jahre zuvor in Italien diese ,zusammengesetzten Opern angeblich genannt wurden. In Italien selbst finden wir diese Bezeichnung zu dieser Zeit augenscheinlich nicht. Die älteste Verwendung des Begriffs findet sich wahrscheinlich im Stück Il Pasticcio, das im Jahre 1759 in Venedig aufgeführt wurde.

Hier soll versucht werden, verschiedene Phänomene aus dem Bereich des Musiktheaters, für die man den Begriff Pasticcio verwendet, zusammenzufassen:

1. Die Kreation einer vollkommen neuen Oper aus Arien verschiedener Komponisten, also ein Pasticcio im ursprünglichen Sinne des Wortes nach Quantz. In diesem Typus sind beide Bedingungen, mit denen das Pasticcio definiert werden kann, vorhanden: a) die Verwendung eines vorher existierenden Materials, b) die Übernahme der Musik verschiedener Autoren (wobei deren Anzahl nicht festge-

6 Als eines der Schlüsselereignisse kann man die Konferenz Operatic Pasticcios in 18th Century Central Europe nennen, die vom 4. bis zum 6. Oktober 2018 in Mainz veranstaltet wurde und deren sich in Vorbereitung befindender Sammelband berechtigte Erwartungen weckt. Von den früheren wissenschaftlichen Konferenzen wäre insbesondere Responsabilità d'autore e collaborazione nell'opera dell'età barocca: Il Pasticcio (Reggio Calabria 2009) anzuführen. 
legt ist). Für diesen Pasticcio-Typ ist eine deutliche Diversität persönlicher Kompositionsstile kennzeichnend. Gleichzeitig ist es hier nicht möglich, das Verdienst des Bearbeiters als Urheber außer Acht zu lassen. Einerseits war er dafür verantwortlich, geeignete Arien auszuwählen und dramaturgisch aufzubauen, andererseits musste er Rezitative und manchmal auch einige Teile der Musiknummern neu vertonen.

2. Die Bearbeitung eines schon existierenden Stückes durch das Einlegen fremder Arien, also „opera impasticciata“ (POLIN 2011). Die Oper war in der Barockzeit ein recht offenes Werk. Bei jedem weiteren Einstudieren ging es nämlich im Grunde genommen um eine neue Version. Bei Reprisen war es üblich, die bestehenden Arien einer neuen Sängerbesetzung, ihren Stimmlagen sowie ihren technischen Fähigkeiten anzupassen. Da der ursprüngliche Komponist meistens nicht an der Neueinstudierung beteiligt war, wurden diese Änderungen nur selten autorisiert. Es ist also verständlich, dass es oft zu einer ,Kontamination“ mit Musik von fremden Autoren kam. Auch da sind beide angegebenen, das Pasticcio definierenden Bedingungen vorhanden. Im Gegensatz zum Zufälligkeitselement ist hier jedoch die ursprüngliche schöpferische Leistung des Urhebers oder der dramaturgische Plan eines originalen Musikwerks nachvollziehbar.

3. Der Rückgriff auf die schon vorhandene eigene Musik, also „self-pasticcio“ (STROHM 1997: 73). Eine solche Komposition bevorzugte insbesondere Vivaldi, der seine eigenen erfolgreichen Arien wiederholt in verschiedenen Opern wieder aufgriff, und zwar auch im Zeitabstand von mehreren Jahren.

4. Ein aufgrund der Kooperation von mehreren Autoren entstandenes Werk, wobei jeder der Komponisten zum Beispiel nur einen Akt vertonte. $\mathrm{Zu}$ den am häufigsten erwähnten Beispielen dieses Pasticcio-Typs gehört die Oper Muzio Scevola der Autoren Filippo Amadei, Giovanni Bononcini und G. F. Händel (London 1721). Die beiden zuletzt genannten Pasticcio-Typen kann man nur mit Vorbehalt als Pasticci sehen - bei ersterem ist die Bedingung der mehrfachen Autorschaft nicht erfüllt und beim zweiten wurde keine vorher bestehende $\mathrm{Mu}$ sik verwendet.

Es ist natürlich möglich, alle diese vier erwähnten Typen noch näher zu präzisieren und in verschiedenen Kontexten zu betrachten. In der typologischen Darlegung wird zum Beispiel die Autorschaft des Texts völlig außer Acht gelassen. Gerade im Bereich der Musikadaption und Neubearbeitung ist auch ein gewisses $\mathrm{Maß}$ an eigener kompositorischer Zugabe in Betracht zu ziehen. Es sollen also im Folgenden zumindest die wichtigsten Richtungen skizziert werden, die die Pasticcio-Forschung einschlagen kann.

Den ersten Bereich stellt die Motivation für das Schaffen eines Pasticcios dar. In Aufsätzen über die Barockoper werden als Hauptgrund für das Entstehen von Pasticci am häufigsten die strikten Vorgaben der Stars unter den Sängerinnen und Sängern erwähnt. Gastierende Virtuosen bestanden auf der Eingliederung ihrer Lieblings-Arien, ohne Rücksicht auf das vorzuführende Stück (man spricht hier von einer sog. ,aria 
di baule', also wörtlich ,Koffer-Arie $\left.{ }^{6}\right)^{7}$ Es ist allerdings offensichtlich, dass ein solches ,Recycling durchaus auch positive Seiten aufwies - die Einstudierung wurde dadurch nicht nur schneller, sondern auch finanziell günstiger. ${ }^{8}$ Weitere Gründe dafür sind auf der betriebspraktischen Ebene zu sehen, und zwar sowohl in sängerischer als auch in instrumentaler Hinsicht (zur damaligen Zeit war die Besetzung von Opernorchestern noch bei Weitem nicht standardisiert; es konnte also vorkommen, dass einige in der ursprünglichen Partitur verlangte Instrumente einfach nicht zur Verfügung standen). Daneben kann man ein musikalisches Prestige-Denken als Motiv für das Schaffen von Pasticci nennen (etwa das Bestreben, neue Musik lokaler Komponisten vorzuführen) oder kommerzielle Absichten (v.a. die Notwendigkeit, dem Geschmack des Publikums zu entsprechen).

Ein weiterer Bereich betrifft die einzelnen Elemente, aus denen Pasticci zusammengesetzt sind, also die Arien. Bei den Arien kann man ihre Wege quer durch einzelne musikdramatische Werke verfolgen, ihre Beziehungen zu Interpreten untersuchen usw. Dabei stellt man oft mit Überraschung fest, dass die klassische ,aria di baule' (d.h. eine von einem konkreten Interpreten in verschiedenen Opern gesungene Arie) nur eine der vielen Erscheinungen der sog. wandernden Arie (wandering aria) ist. Bei einer neu in ein schon bestehendes Musikwerk eingelegten Arie, also einer Einlagearie (inserted aria), können die ursprüngliche Oper (entlehnte Arie - borrowed aria) oder weitere Fälle von Präsentationen (gemeinsam verwendete Arie - shared aria) identifiziert werden. Auch die Beziehung eines Sängers zu einer konkreten Musiknummer, die bei der ursprünglichen Einstudierung nicht unbedingt ein Bestandteil seiner Rolle sein musste, sondern durch eine andere Figur interpretiert werden konnte, ist interessant (gestohlene Arie - stolen aria).

Eingehendere Untersuchungen bedarf bei den Wanderarien auch das Problem der Neubearbeitung der Texte, v.a. die Anwendung von Parodie- oder Kontrafaktur-Techniken (FALCK 1979). Grundsätzlich gab es zwei Arten der Arbeit mit dem Text. Am häufigsten kam jene zum Einsatz, bei der man die Arie mehr oder weniger komplex, also einschließlich des Textes, übernahm und nur einige Wörter oder Verse entsprechend der neuen dramatischen Situation adaptiert wurden. Schwerwiegender war dagegen das Unterlegen des Ziellibretto-Textes, da es eine umfangreichere Musikbearbeitung erforderlich machte. In diesem Fall wird ein ganzer Komplex von Fragen angeschnitten, wie die Übereinstimmung der Textbedeutung mit den Regeln der barocken musikalischen Rhetorik, der Abgleich von Musik- und Wortakzent usw.

7 Diese Praxis wird auch mit zahlreichen Beispielen aus konkreten Quellen belegt; so verhielt es sich auch im 19. Jahrhundert, siehe (PORISS 2009).

8 Es gibt sogar Belege dafür, dass man einem Komponisten von Pasticci genauso viel wie für eine neu komponierte Oper bezahlte (STROHM 1985: 165). 


\section{Institutionen: die städtische Oper und private Adelsbühnen}

Die italienische Oper kommt grundsätzlich Mitte der Zwanzigerjahre des 18. Jahrhunderts in die böhmischen Länder, auch wenn es vereinzelte Belege für frühere Aufführungen gibt. ${ }^{9}$ Als wichtigster Impuls gilt die Inszenierung der monumentalen ,festa teatrale 'Costanza e fortezza anlässlich der Krönung von Karl VI. in Prag im Jahre $1723 .{ }^{10}$ Im Jahre $1724 \mathrm{kam}$ eine Theatergesellschaft aus Venedig, geleitet von Antonio Maria Peruzzi und Antonio Denzio, nach Böhmen und nahm ihren Opernbetrieb am Prager Theater im Palast des Grafen Franz Anton Sporck auf (FREEMAN 1992). Ein Jahr später begann ein separater Teil der Prager Gesellschaft in Breslau seine Tätigkeit. Hier fanden bis zum Jahre 1739 Opernvorstellungen statt. Die wichtigste Persönlichkeit der schlesischen Opernszene war der Komponist und Impresario Antonio Bioni (BORCHERT 1910; SPÁČILOVÁ 2016b). In den Jahren 1732-1740 führte man im Brünner städtischen Theater, Theater in der Taverne, Opern auf, wobei Angelo Mingotti von den hier tätigen Direktoren der bedeutendste war (HAVLÍČKOVÁ 2012).

Zur gleichen Zeit wie in die Hauptstadt des Königreichs Böhmen hielt die italienische Oper auch in mährische Schlösser Einzug. Aus dem Jahre 1723 stammen die ersten Berichte über Opernaufführungen in Jaroměřice nad Rokytnou / Jaromeritz. Graf Johann Adam Questenberg, ein bedeutender Musikliebhaber und Förderer, hielt sich hier einige Jahre lang ein fixes künstlerisches Ensemble aus dem Kreis seiner Untertanen mit dem Kapellmeister Franz Anton Mitscha an der Spitze (PERUTKOVÁ 2015). In den Jahren 1727-1737 ließ der Olmützer Bischof Wolfgang Hannibal Schrattenbach in seinen Schlössern in Kroměřǐž / Kremsier und in Vyškov / Wischau Opern aufführen (SPÁČILOVÁ 2018a). Das letzte mährische Zentrum der italienischen Oper war in den Jahren 1733-1739 Holešov / Holleschau, Sitz des Grafen Franz Anton Rottal (JURÁŠKOVÁ und SPÁČILOVÁ 2019). Freilich ist es angebracht, die angeführten Ortschaften im Kontext etablierter Zentren der italienischen Oper wie Wien oder Dresden zu sehen.

Alle genannten Zentren der italienischen Oper waren personell eng verknüpft. Eine Operngesellschaft bestand zu dieser Zeit in der Regel aus dem Kapellmeister und Komponisten in einer Person, einem Orchesterleiter (dem ersten Geigenspieler) und manchmal auch einem Theaterbildner oder einem Ballettmeister sowie aus 6 bis 8 Sängern, die für eine oder mehrere Saisonen engagiert waren. Die Schicksale einzelner Mitglieder dieser Gesellschaften, die den Libretti, den Korrespondenzen und einigen wenigen Vermerken in diversen Matrikeln zu entnehmen sind, zeigen, dass ein italienischer Künstler - falls er es sich zutraute, sein Glück in den Ländern jenseits der Alpen zu suchen - dort meistens mehrere Saisonen blieb oder üblicherweise mehrfach zurückkehrte. Das war auch der Fall bei Matteo Lucchini aus Venedig, der als Sänger

9 Abgesehen von den Gastvorstellungen der Wiener Hofkapelle bei Besuchen habsburgischer Kaiser in den böhmischen Ländern waren es insbesondere die in Prag in den Jahren 1702-1703 aufgeführten Opern der Gesellschaft von Giovanni Federico Sartorio sowie die Serenate Piramo e Tisbe, deren Librettodruck in Olmütz im Jahre 1712 erschien.

$10 \mathrm{Zu}$ dieser außergewöhnlichen Veranstaltung siehe zusammenfassend (VÁCHA et al. 2009). 
und Komponist in Dresden (1725), Prag (1726-1730, 1734-1735), Breslau (1731-1733) und Brünn (1736-1740) tätig war. Als ein weiteres Beispiel ist Giuseppe Nicola Alberti zu nennen, der zwar nach seiner Tätigkeit in Prag und Breslau (1725-1729) nach Italien zurückkehrte, sich jedoch im Jahre 1733 erneut auf den Weg über die Alpen machte. Neben seinem Engagement als Sänger in Brünn erwarb er auch den Titel „Directore der Operen und Music“ des Grafen Rottal in Holleschau (1733-1736). Die Ensembles im aristokratischen Umfeld waren in Personalangelegenheiten autonom, doch auch hier konnten gastierende Künstler erfolgreich tätig sein. Das gilt vor allem für die schon erwähnte Stadt Holleschau, wo italienische Künstler, die in Brünn ein Engagement hatten, und im Jahre 1737 sogar Solisten der kaiserlichen Hofkapelle und des Wiener Kärntnertortheaters regelmäßig aufzutreten pflegten (SPÁČILOVÁ 2016a; PERUTKOVÁ 2016). Das Kommunikationsnetz von mehreren Dutzend wandernden Künstlern hatte natürlich einen deutlichen Einfluss auf die Zirkulation des Repertoires.

\section{Quellen: Libretti und Partituren}

Im Umkreis der böhmischen Länder sind wir in der Forschung zur italienischen Barockoper fast ausschließlich auf gedruckte Libretti angewiesen, die für jede Einstudierung neu herausgegeben wurden. Heute kann man mit mehr als 150 Libretti arbeiten, die oft in verschiedenen Bibliotheken der Welt verstreut erhalten geblieben sind. ${ }^{11}$ Bei den Musikmaterialien ist eine unverhältnismäßig geringere Anzahl überliefert, genauer gesagt handelt es sich um etwa ein Dutzend erhalten gebliebener Opernpartituren aus Jaromeritz und Breslau. ${ }^{12}$

Auf die Verwendung von Musik mehrerer Komponisten macht in diesen Quellen eine breite Skala von verschiedenen Indizien aufmerksam, ob es sich nun um neu geschaffene Pasticci oder mehr oder weniger bearbeitete Originalopern handelt. Das üblichste Zeichen, mit dem in Libretti italienischer Provenienz die Einlagearie bezeichnet wird, die ,stelletta' (ein kleines Sternchen) findet man in dem zu erforschenden Umfeld nur sehr selten. Als Beispiel dafür kann die Oper Partenope von Eustachio Bambini dienen (Holleschau 1733), wo die eingelegten Arien der lokalen Komponisten Johann Georg Orschler und Ferdinand Seidl mit Asterisken versehen wurden. Viel üblicher war es, die Angabe über die musikalische Autorschaft mit einer lakonischen Formel zu ergänzen, die „mit Ausnahme von einigen nach dem Wunsch von Solisten eingelegten Arien" lautete, wie sie in fast allen aus Brünn und in einigen aus Prag kommenden Libretti vorkommt. Eine besondere Lösung der mehrfachen Autorschaft ist zum Beispiel in dem Libretto La costanza di Griselda (Breslau 1728) zu sehen, wo bei jeder Arie der Name des verantwortlichen Komponisten eigens geschrieben steht. Als Kuriositäten sind dabei ,makkaronische“ Arien, also Passagen in Nationalsprachen, die

11 Aus der Zeit vor dem Jahr 1750 sind mehr als 60 Libretti aus Prag, 27 Libretti aus Breslau und 81 aus Mähren (Brünn, Jaromeritz, Kremsier, Wischau, Holleschau) bekannt (SPÁČILOVÁ, in Vorbereitung [b]).

12 Die Zahl von Partituren aus der Questenberg-Sammlung erreicht knapp 50 Stück, doch nur für einen Teil davon kann man auch die Aufführung des Werks belegen (PERUTKOVÁ 2015: 557-570). 
in italienische Opern eingelegt wurden, zu erwähnen. Ein Beispiel für die Verwendung der deutschen Sprache sind die in Bionis Serenate Endimione eingelegten Arien von Daniel Gottlieb Treu. Tschechisch kam in der anonymen Oper Il confronto dell'amor coniugale (Prag 1727) vor. ${ }^{13}$

Doch auch in Fällen, in denen die Quelle selbst auf keine Art und Weise die Verwendung einer fremden Musik andeutet, kann man durch eine Textanalyse zum Ergebnis kommen, dass es sich um ein Pasticcio handelt. In dieser Hinsicht sind Libretti aus Kremsier und Wischau besonders interessant, in denen das Bestreben sichtbar ist, die Texte der eingelegten Arien möglichst der Bedeutung des Zielstücks anzunähern. Wenn also eine neue Arie mit einem abweichenden Versschema verwendet wurde, war diese oft durchaus kreativ, doch unter Verwendung derselben Begrifflichkeit nachgedichtet, um eine Beschädigung des dramatischen Textaufbaus zu verhindern (SPÁČILOVÁ 2018a: 135-138).

Musikmaterialien umfassen natürlich ein viel breiteres Spektrum an Angaben als Libretti. Als die interessantesten gelten ,Arbeitspartituren', in die die Einlagearien direkt eingenäht oder eingeklebt wurden. Wertvolle Informationen über den Entstehungsprozess eines neuen Musikstücks sind ferner auch Streichungen und umgeschriebenen Passagen zu entnehmen, wo die ursprüngliche Schicht der Eintragung ausgekratzt oder überklebt werden konnte (doch nicht musste). Ein direkter Hinweis auf die Provenienz ist keinesfalls die Regel, insofern stellt eine mit der Inschrift „Napoli 1737 del signore Leonardo Leo" versehene Arie, die in die Partitur von Demofoonte (Jaromeritz 1738) von Giuseppe Ferdinando Brivio eingelegt wurde, ${ }^{14}$ eine echte Ausnahme dar, oder aber auch die Bezeichnung der Autoren neuer Arien in der Partitur von Orlandinis Oper Antigona (Breslau 1728) (SPÁČILOVÁ 2018b).

Man könnte erwarten, dass das Pasticcio-Prinzip nur im Bereich städtischer Bühnen im größeren Maßstab Anwendung fand, und zwar einerseits wegen der Eingliederung von wandernden Solisten mit ihren ,arie di baule und andererseits wegen einer größeren Abhängigkeit von der Gunst des Publikums, die für die Impresarien von existenzieller Bedeutung war. Doch tatsächlich ist das Gegenteil der Fall. Einer ausführlicheren Quellenforschung ist zu entnehmen, dass fremde Musik in gleichem Maße auch in die auf privaten Adelsbühnen aufgeführten Opern Einzug hielt. Es ist unwahrscheinlich, dass nur praktische Gründe zu solchen Eingriffen geführt haben, da die gesanglichen Fähigkeiten der lokalen Sänger, so ist anzunehmen, auf einem sehr hohen Niveau waren. Vermutlich gehen umfangreiche Bearbeitungen eher auf das Bestreben zurück, die bestehenden Stücke zu modernisieren bzw. neu zu beleben oder - im Falle neuer Pasticci - der Vorstellung der Zuschauer von italienischer Oper zu entsprechen.

In diesem Sinne lässt sich jedenfalls auch eine wertvolle zeitgenössische Quelle, der Breslauer Schlesische Nouvellen-Courier, vernehmen. In den regelmäßigen Nachrichten über das dortige Theater wurden Reprisen von Opern mit neuen Arien, die als

13 Messalinas Arie „Gsme vesselj a spiwáme“, interpretiert von der venezianischen Sängerin Teresia Peruzzi, war vermutlich die erste auf Tschechisch gesungene Opernarie (FREEMAN 1992: 124-125).

14 Die Arie stammt aus der Oper Farnace von Leonardo Leo, aufgeführt tatsächlich in Neapel im Jahre 1737 (PERUTKOVÁ 2015: 487). 
ein Mittel zur Wiederbelebung des eingeschlafenen Publikumsinteresses dienen sollten (wörtlich „um das Publicum mit der Diversitaet zu bedienen“), annonciert. Eine hohe Wertschätzung genossen auch neue, originale, aus Arien der besten italienischen Meister zusammengesetzte Pasticci, bei denen die „Differenz im Gusto“ betont wurde (SPÁČILOVÁ 2017: 164). Daraus kann man schließen, dass das Einlegen einer fremden Musik aus der damaligen Sicht nicht als Wertminderung, sondern als Bereicherung des bestehenden Werks angesehen wurde.

\section{Erweiterungen: Oratorium, lateinisches Ordenstheater}

Das Pasticcio-Prinzip wurde in dem hier untersuchten Zeitraum in den böhmischen Ländern insoweit erweitert, als es neben der Oper auch weitere dramatische Gattungen, namentlich das Oratorium und das lateinische Ordenstheater, betraf. Die Bereicherung der Oratorien durch Opernarien kann man mit zwei Beispielen aus dem Bereich der Hofkapelle des schon erwähnten Olmützer Bischofs Schrattenbach belegen. In der Fastenzeit, in der Opernaufführungen verboten waren, wurden in seinem Palast in Brünn jede Woche italienische Oratorien gespielt. ${ }^{15}$ Bedauerlicherweise blieben von dieser reichen musikalischen Tradition nur wenige Partituren erhalten, von denen aber die meisten Einlagearien beinhalten, wobei es gelungen ist, einen Teil davon zu identifizieren. In der Partitur zum Oratorium Morte e sepoltura di Christo von Antonio Caldara (Brünn 1730) findet sich eine Arie aus der Oper Siface von Nicola Porpora, in dem Oratorium Cristo nell'orto von Johann Joseph Fux (Brünn 1731) gibt es gleich mehrere Arien aus Geminiano Giacomellis Scipione in Cartagine nuova (Piacenza 1730) und eine Arie aus Alessandro nell'Indie von Leonardo Vinci (Rom 1730). Beim zweiten der genannten Beispiele ist insbesondere der kurze Zeitabstand zwischen den italienischen Premieren der Opern und der Verwendung von Arien daraus in Mähren bemerkenswert. Eine umgekehrte Vorgehensweise - also das Einlegen von Oratorien-Arien in Opern - kann man mit einem Beispiel von zwei Arien aus Porporas Il martirio di S. Giovanni Nepomuceno (Brünn 1732) in der Oper Nitocri, aufgeführt im Jahre 1735 in Wischau, belegen (SPÁČILOVÁ 2018a: 174-183).

Das älteste komplette Oratorium-Pasticcio in den Ländern der böhmischen Krone ist das lateinische Oratorium Fides, Spes, Charitas, aufgeführt in der Kirche der Prager Kreuzherren mit dem Roten Stern am Karfreitag 1725. Dieses Pasticcio setzte das Ordensmitglied Karl Kořínek aus umgetexteten Opernarien Händels zusammen (SPÁČILOVÁ 2019). Ein weiterer Titel ist zum Beispiel das Oratorium La vittima d'amore, geschrieben im Jahre 1741 in Brünn von dem Dietrichsteiner Kapellmeister Joseph Umstatt unter Verwendung verschiedener Arien von Caldara, Johann Adolf Hasse, Leonardo Leo, Francesco Feo, Giovanni Porta und Giovanni Battista Pescetti, und zwar

15 Die Berichte über die Oratorien stammen aus den Jahren 1723-1738, von Libretti sind 36 Titeln bekannt. 
teilweise einschließlich der Texte (VOSS 2018). ${ }^{16}$ Als kontrafaktierte (also umgetextete) Pasticci entstanden allerdings recht viele der Oratorien, die in den 1730er Jahren unter Schrattenbach aufgeführt wurden. Gegen Mitte des 18. Jahrhunderts verfasste auch der Regenschori der Prager Kreuzherren, Josef Bleylebel, nach diesem Prinzip mehrere Oratorien.

Das letzte - etwas kuriose - Beispiel stammt aus dem Bereich des Ordenstheaters und ist eine lateinische, 1738 im Zisterzienserkloster Osek / Ossegg in Nordböhmen unter dem Namen Facetum musicum aufgeführte Karnevaloper. Vier Epikureer werden hier von Freiheit (Libertas) zur mutwilligen Unterhaltung aufgefordert, doch der Philosoph Diogenes rät ihnen von ihrer Ausgelassenheit ab. Die musikalische Komponente besteht aus Arien von Händel, Vivaldi und Lotti, wobei interessant ist, dass es sich in diesem Fall um ein vergleichsweise ,altmodisches' Musikmaterial handelt - alle nachgewiesenen Opern stammen aus dem ersten Jahrzehnt des 18. Jahrhunderts (SPÁČILOVÁ und MACEK 2011).

Angesichts dieser Fakten muss man zugeben, dass unsere neuzeitlichen Vorstellungen von ,geistlicher" und, weltlicher" Musik in Bezug auf die barocke Praxis vollkommen unmaßgeblich sind. Die Oper hielt im Übrigen immer wieder Einzug in das kirchliche Milieu, wie auch die lateinischen Kontrafakturen von Opernarien, die als Kirchenmotetten, am häufigsten in Offertorien zum Einsatz kamen, belegen (JONÁŠOVÁ 2008). Dass das Pasticcio-Prinzip quer durch die musikdramatischen Gattungen festzustellen ist, stellt allerdings einen weiteren Beleg für seine Eigenständigkeit und Relevanz dar.

\section{Schlussfolgerungen}

Es ist sicherlich nicht möglich, die Ursachen für die Beliebtheit des Pasticcios in den böhmischen Ländern restlos zu klären. Dessen ungeachtet lassen sich jedoch einige Thesen aufstellen, die bei der Beantwortung dieser wirklich nicht einfachen Frage helfen könnten. Gab bei der Verwendung fremder Musik tatsächlich nur die Unfähigkeit lokaler Komponisten den Ausschlag, ein komplexes musikdramatisches, den Kriterien für die zeitgenössische Oper oder für ein Oratorium entsprechendes Werk zu schaffen? Das ist bestimmt nicht der Fall. Eher scheint es so zu sein, dass das Publikum ein solches schöpferisches Vorhaben einfach nicht verlangte. Die italienische Oper wurde in den Ländern der böhmischen Krone sehr positiv aufgenommen und die Art und Weise, neue Werke auf der Basis einer vorher schon existierenden Musik zu schaffen, wurde nicht nur als vollkommen legitim, sondern auch als ästhetisch wertvoll betrachtet. Auch praktische Aspekte der Zirkulation von Musikalien spielten bestimmt eine bedeutende Rolle. Denn es war einfacher, mit Abschriften einzelner Arien als mit Abschriften ganzer Partituren umzugehen (und es war auch unverhältnismäßig günstiger, die erstgenannten zu erwerben). Die Variationsmöglichkeiten und neuen

16 Eine Reprise des Werkes gab es auch im Jahre 1744 in Prag, die Partitur wurde den Kreuzherren von dem damaligen Rottal- und Dietrichstein-Tenoristen Karl Beer zur Verfügung gestellt. 
Kontextualisierungen einzelner Teile dieser musikalischen „Puzzles' waren sozusagen unendlich, und wenn man bei unseren Vorfahren von der gleichen Vorliebe für die spielerische Kreativität und Improvisation ausgehen kann, die für die Einwohner der böhmischen Länder auch in unseren Tagen noch kennzeichnend ist, sind wir bestimmt nicht weit entfernt, einen Schlüssel für das Verständnis dieses Phänomens zu finden.

Übersetzung: Eva Kušová, Christian Neuhuber.

\section{Bibliografie}

BORCHERDT, Hans Heinrich. 1910. Geschichte der italienischen Oper in Breslau. Zeitschrift des Vereins für Geschichte Schlesiens 44 (1910): 18-51.

FALCK, Robert. 1979. Parody and Contrafactum: A Terminological Clarification. The Musical Quarterly 65 (1979): 1: 1-21.

FREEMAN, Daniel E. 1992. The Opera Theater of Count Franz Anton von Sporck in Prague. Studies in Czech Music, No. 2. New York: Pendragon Press, 1992.

HAVLÍČKOVÁ, Margita. 2012. Berufstheater in Brünn 1668-1733. Brno: Masarykova univerzita, 2012.

JONÁŠOVÁ, Milada. 2008. Italienische Opernarien im Dom zu St. Veit in Prag. In Corinna Herr, Herbert Seifert, Andrea Sommer-Mathis und Reinhard Strohm (Hg.). Italian Opera in Central Europe 1614-1780. Volume 2: Italianità: Image and Practice. Berlin: Berliner Wissenschafts-Verlag, 2008: 163-206.

JURÁŠKKOVÁ, Kateřina und Jana SPÁČILOVÁ. 2019. Italská opera na holešovském zámku v době Františka Antonina Rottala. Holešov: Město Holešov, 2019.

LAZAREVICH, Gordana. 1976. Eighteenth-Century Pasticcio: the Historian's Gordian Knot. Analecta Musicologica 17 (1976): 121-145.

PERUTKOVÁ, Jana. 2015. Der glorreiche Nahmen Adami. Johann Adam Graf von Questenberg (16781752) als Förderer der italienischen Oper in Mähren. Wien: Hollitzer, 2015.

PERUTKOVÁ, Jana. 2016. Vienna Kärntnertortheater Singers in the Letters from Georg Adam Hoffmann to Count Johann Adam von Questenberg. Italian Opera Singers in Moravian Sources c. 1720-1740 (Part II). In Gesa zur Nieden und Berthold Over (Hg.). Musicians' Mobilities and Music Migrations in Early Modern Europe. Biographical Patterns and Cultural Exchanges. Mainz Historical Cultural Sciences 33, Bielefeld: Transcript, 2016: 275-292.

POLIN, Giovanni. 2011. Le „opere che al dosso degli attor non son tagliate riescon per ordinario impasticciate“. Riflessioni sullo status del testo spettacolare melodrammatico nel '700. In Gaetano Pitarresi (Hg.). Responsabilità d'autore e collaborazione nell'opera dell'Età barocca: Il Pasticcio, Atti del convegno internazionale di studi. Reggio Calabria: Laruffa Editore, 2011: 325-364.

PORISS Hilary. 2009. Changing the Score: Arias, Prima Donnas, and the Authority of Performance. Oxford: Oxford University Press, 2009.

QUANTZ, Johann Joachim. 1755. Herrn Johann Joachim Quantzens Lebenslauf, von ihm selbst entworfen. In Friedrich Wilhelm Marpurg (Hg.). Historisch-Kritische Beyträge zur Aufnahme der Musik 1 (1755): 3: 197-250. 
SARTORI, Claudio. 1990-1994. I libretti italiani a stampa dalle origini al 1800. Catalogo analitico con 16 indici. Cuneo: Bertola e Locatelli, 1990-1994.

SIEGERT, Christine. 2016. Zum Pasticcio-Problem. In Thomas Betzwieser (Hg.). Opernkonzeptionen zwischen Berlin und Bayreuth. Das musikalische Theater der Markgräfin Wilhelmine, Thurnauer Schriften zum Musiktheater 31. Würzburg: Königshausen und Neumann, 2016: 155-166.

SPÁČILOVÁ, Jana. 2011. Libretto as a Source of Baroque Scenography in the Czech Lands. In Christian M. Billing und Pavel Drábek (Hg.). Czech Stage Art and Stage Design. Special Issue of Theatralia/Yorick 1 (2011): 82-97.

SPÁČILOVÁ, Jana. 2016a. Soloists of the Opera Productions in Brno, Holešov, Kroměříz and Vyškov. Italian Opera Singers in Moravian Sources c. 1720-1740 (Part I). In Gesa zur Nieden und Berthold Over (Hg.). Musicians' Mobilities and Music Migrations in Early Modern Europe. Biographical Patterns and Cultural Exchanges. Mainz Historical Cultural Sciences 33, Bielefeld: Transcript, 2016: 255-273.

SPÁČILOVÁ, Jana. 2016b. Počátky opery ve Slezku - současný stav vědomostí. Musicologica Brunensia 51 (2016): 2: 157-170.

SPÁČILOVÁ, Jana. 2018a. Hudba na dvoře olomouckého biskupa Schrattenbacha. Olomouc: FF UP, 2018.

SPÁČILOVÁ, Jana. 2018b. Orlandini's Antigona (vendicata): Transformation of a Venetian Opera on its Transalpine Journey. Musicologica Brunensia 53 (2018): Supplementum: 227-248.

SPÁČILOVÁ, Jana. 2019. Zur Händel-Rezeption in den böhmischen Ländern in der ersten Hälfte des 18. Jahrhunderts. Händel-Jahrbuch 65 (2019): 261-273.

SPÁČILOVÁ, Jana. In Vorbereitung [a]. Local Conditions of Pasticcio Production and Reception: Between Prague, Wrocław and Moravia. In Gesa zur Nieden und Berthold Over (Hg.). Operatic Pasticcios in 18th-Century Europe. Contexts, Materials and Aesthetics. Bielefeld: Transcript, in Vorbereitung.

SPÁČILOVÁ, Jana. In Vorbereitung [b]. Catalogue of the Italian Opera Libretti in Central Europe in the First Half of the Eighteenth Century, I: Moravia. Praha: KLP, in Vorbereitung.

SPÁČILOVÁ, Jana und Ondřej MACEK. 2011. Nová zjištění k latinské karnevalové opeře „Facetum musicum / Musicalisches Kurtzweil-Spiel“ (Osek 1738). Hudebni věda 48 (2011): 2-3: $143-160$.

STROHM, Reinhardt. 1980. Pasticcio. In Stanley Sadie (Hg.). The New Grove Dictionary of Music and Musicians. London: Macmillan, 1980: 14: 288-289.

STROHM, Reinhardt. 1985. Händel's pasticci. In Reinhardt Strohm. Essays on Handel and Italian Opera. Cambridge: Cambridge University Press, 1985: 164-211.

STROHM, Reinhard. 1997. Dramma per Musica. Italian Opera Seria of the Eighteenth Century. New Haven/London: Yale University Press, 1997.

STROHM, Reinhard. 2008. The Operas of Antonio Vivaldi. Firenze: Leo S. Olschki Editore, 2008.

VÁCHA et. al. 2009. Štěpán Vácha, Irena Veselá, Vít Vlnas und Petra Vokáčová. Karel VI. a Alžběta Kristýna. Česká korunovace 1723. Praha: Paseka, 2009.

VOSS, Steffen. 2018. Das Johann Adolf Hasse zugeschriebene Passions-Oratorium La morte di Cristo und seine musikhistorische Einordnung. Musicologica Brunensia 53 (2018): Supplementum: 261-281. 


\section{Mgr. Jana Spáčilová, Ph.D.}

Katedra muzikologie, Filozofická fakulta, Univerzita Palackého v Olomouci, Univerzitní 3, 77180 Olomouc, Česká republika jana.spacilova@upol.cz

Jana Spáčilová studierte an der Karls-Universität Prag Musikwissenschaften und setzte ihr Doktoratsstudium an der Masaryk-Universität Brünn fort. Dort wirkte sie am Institut für Musikwissenschaften zunächst als wissenschaftliche Mitarbeiterin, dann als Assistentin. 20072014 war sie Kuratorin für Alte Musik in der Abteilung Musikgeschichte des Mährischen Landesmuseums. Derzeit lehrt sie am Lehrstuhl für Musikologie der Palacký-Universität Olmütz, seit 2019 als Dozentin. Ihr wissenschaftliches Interesse gilt vor allem der italienischen Oper und dem Oratorium in Mitteleuropa im 18. Jahrhundert sowie der Musik in den böhmischen Ländern zur Zeit des Barock. 